\author{
Military Technical College \\ Kobry Elkobbah, \\ Cairo, Egypt
}

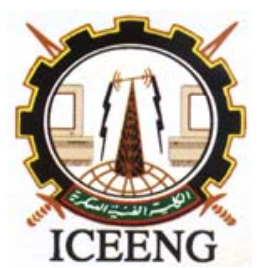

\author{
$5^{\text {th }}$ International Conference \\ on Electrical Engineering \\ ICEENG 2006
}

\title{
AUTOMATIC CLASSIFICATION OF QUADRATURE AMPLITUDE MODULATED (QAM) SIGNALS IN PRESENCE OF CHANNEL IMPAIRMENTS
}

\author{
Tarek Helaly $^{*} \&$ Khairy Elbarbary ${ }^{* *}$
}

\begin{abstract}
In this paper, a procedure for automatic classification among three types of QAM signals in presence of channel impairments is proposed. A combination of digital signal processing and pattern recognition methods is used for solving the classification problem. The proposed algorithm has been tested by computer simulations and has proven to be reliable and robust against the expected channel impairments such as noise and fading. It is found that all digital modulation types of interest have been correctly classified with a success rate $>91 \%$ at signal-to-noise ratio (SNR) of $14 \mathrm{~dB}$.
\end{abstract}

\section{Key Words}

Signal Processing, Modulation Recognition, and Automatic Signal Classification.

\section{Introduction}

This paper is concerned with automatic modulation classification of digital communication signals. Modulation classification represents an intermediate step between signal interception and demodulation. It has several military and civilian applications. One of the military applications is communication intelligence in electronic warfare which is intended to determine the signature of the enemy transmitters by extracting the parameters that characterize their signals. These parameters are useful in threat recognition by comparing the signal characteristics of intercepted transmitter against a catalogue of characteristics or signal sorting parameters. Another application of modulation classification is that it enables us to produce a smart jamming against the intercepted signals. This is performed by generating a jamming signal with identical characteristics of the intercepted signal that contains false information to confuse the enemy.

* Associate Lecturer, Electronic Warfare Eng. Department, MTC, Cairo, Egypt.

** Associate Professor, Electronic Warfare Eng. Department, MTC, Cairo, Egypt. 
Civilian applications include signal confirmation, interference identification, detection of non-licensed transmitters, and supervision of admitted wireless stations, to determine whether they obey the limits of their operation parameters.

Actually, few articles were published in this area. In [1], a classifier that is based on the artificial neural network is used to classify analogue and digital modulated signals. Several key features are extracted from the received signals and are used in the classification. In [2], a classifier that is based on pattern recognition method is described. The power spectrum is applied as a signal feature that can be used in the classification. The classification algorithm is developed as a software application using digital signal processing technique. In [3], a classifier is proposed for digital modulation schemes based on fourth-order cumulants. In [4], the maximum-likelihood (ML) method is used to discriminate between QAM-16, QAM-32, and QAM-64. It is shown that the ML classifier is capable of classifying any finite set of distinctive constellations with zero error rates when the number of available data symbols goes to infinity. In [5], a classifier for MFSK signals contaminated with class-A impulsive noise and transmitted over time varying flat correlated fading channel is developed.

The proposed algorithm in this paper utilizes the statistical pattern recognition approach for the classification among three types of QAM signals (QAMI, QAMII, and QAMIII). Table 1 shows both the amplitude and phase characteristics of these types of modulations. These characteristics are such that for each type of QAM a certain number of amplitude and phase levels are assigned. The signal constellations of each modulation type of interest are shown in Fig. 1. The proposed algorithm is based on both the amplitude and phase characteristics of the intercepted signal.

Table 1. Amplitude and Phase characteristics of QAM-16 types

\begin{tabular}{|c|c|c||}
\hline Type & Number of amplitude levels & Number of phase levels \\
\hline QAM I & 2 & 8 \\
\hline QAM II & 3 & 12 \\
\hline QAM III & 4 & 8 \\
\hline
\end{tabular}

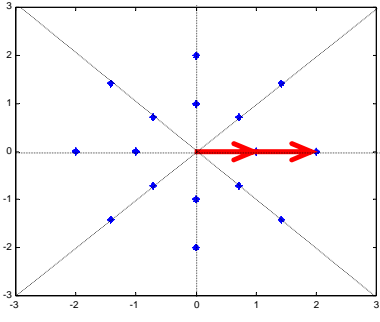

(a)

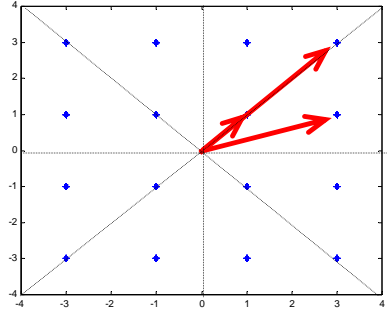

(b)

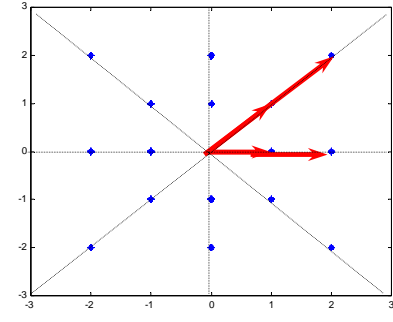

(c)

Fig. 1 Signal constellations of (a) QAM I, (b) QAM II, and (c) QAM III 
This paper consists of four main sections. In section 2, the signal and channel model is presented. In section 3, the proposed algorithm is introduced. In section 4, thresholds determination and performance evaluation for the proposed classifier are shown. Finally, the conclusion is presented in section 5 .

\section{Signal and channel model}

The modulated signal, $s(t)$, can be written as:

$$
s(t)=\operatorname{Re}\left\{A(t)\left[e^{j\left(\omega_{c} t+\theta(t)\right)}\right]\right\}
$$

where $A(t)$ is the signal amplitude, $\omega_{c}$ is the carrier angular frequency, and $\theta(t)$ is the signal phase. The signal, $s(t)$, is transmitted over a flat (frequency non-selective) fading channel in which its coherence bandwidth is much greater than the signal bandwidth. The channel fading effect, $F$, can be written as [6]:

$$
F=\eta e^{-j \theta_{F}}
$$

where $\eta$ is the envelope of the multi-path signal which has a Rayleigh distribution with probability density function given by [6]

$$
f(\eta)=\frac{2 \eta}{b_{o}} \exp \left(\frac{\eta^{2}}{b_{o}}\right) ; \eta>0
$$

where $b_{o}$ represents the average scattered power due to multi-path. The phase $\theta_{F}$ has a uniform distribution and is given by [6]

$$
f_{\Theta_{F}}\left(\theta_{F}\right)=\frac{1}{2 \pi} ; 0 \leq \theta_{F} \leq 2 \pi
$$

The additive noise, $w(t)$, is assumed to be white Gaussian noise with power spectral density of $N_{0} / 2$. Thus received signal, $r(t)$, has the form

$$
\begin{aligned}
r(t) & =F s(t)+w(t) \\
& =\operatorname{Re}\left\{\eta A(t)\left[e^{j\left(\omega_{c} t+\theta(t)-\theta_{F}\right)}\right]\right\}+w(t)
\end{aligned}
$$

\section{Proposed Classifier}

The proposed classifier consists of three main stages; 1) Preprocessing stage, 2) key features extraction stage, and 3) Classification stage. The structure of the proposed classifier is depicted in Fig. 2. In the following each stage will be explained in details. 


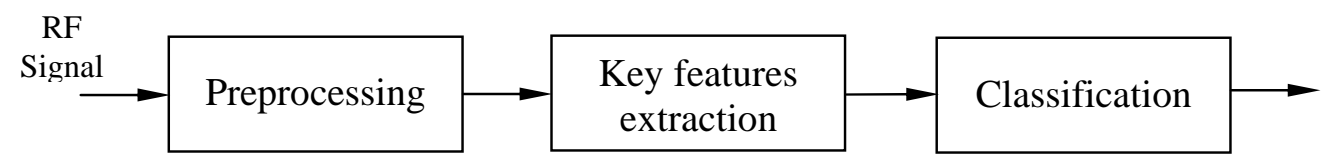

Fig.2 Structure of the proposed

\section{A) Preprocessing stage}

In this stage, the instantaneous phase and amplitude of the received signal are computed. The signal $r(t)$ contains phase and amplitude information that can be extracted by means of Inphase-Quadrature (IQ) techniques or by using discrete Hilbert transform [7]. The extracted instantaneous phase can be represented as

$$
\varphi(t)=\theta(t)-\theta_{F}-\theta_{w}(t) ;-\pi \leq \varphi(t)<\pi
$$

where $\theta_{w}(t)$ is the random phase attributed to the noise. The extracted instantaneous amplitude can be represented as [7]

$$
a(t)=\sqrt{\eta^{2} A^{2}(t)+|w(t)|^{2}+2 \eta A(t)|w(t)| \cos \left(\theta(t)-\theta_{F}-\theta_{w}(t)\right)}
$$

where $|w(t)|$ is the magnitude of the additive noise at the classifier .

\section{B) Key features extraction stage}

In this stage, we evaluate two key features, which are used in the classification process. The first one is the normalized histogram of instantaneous phase of the received signal while the second one is the normalized histogram of instantaneous amplitude of the received signal.

In general, the histogram of a received signal parameter, $\psi$, is a discrete approximation to the probability density function (pdf) of the received parameter [4], that is

$$
H_{\Psi}(\psi)=\frac{F_{\Psi}(\psi(i))-F_{\Psi}(\psi(i-1))}{\psi(i)-\psi(i-1)} ; \psi(i-1) \leq \psi<\psi(i), \quad i=1, \ldots ., k
$$

where $k$ is the number of subintervals and $F_{\Psi}(\psi)$ is the cumulative distribution function of the received parameter, $\psi$, and it is calculated as

$$
F_{\Psi}(\psi)=\int_{-\infty}^{\psi} f_{\Psi}(y) d y
$$

where $f_{\Psi}(y)$ is the pdf of the parameter, $\psi$.

Using the above definition, the histogram of instantaneous phase of the received signal, $H_{\Phi}(\varphi(i))$, is evaluated over 48 subintervals in the [- $\pi, \pi$ [ interval. The choice of the 48 subintervals is taken in order to achieve a minimum reasonable resolution between any two adjacent states. The normalized phase histogram can be calculated as follows, 


$$
\dot{H}_{\Phi}(\varphi(i))=\frac{H_{\Phi}(\varphi(i))}{\max \left\{H_{\Phi}(\varphi)\right\}}
$$

Similarly, the histogram of the instantaneous amplitude of the received signal, $H_{A}(i)$, is evaluated over 12 subintervals. The normalized amplitude histogram can be calculated as follows,

$$
\dot{H}_{A}(a(i))=\frac{H_{A}(a(i))}{\max \left\{H_{A}(a)\right\}}
$$

\section{C) Classification stage}

In the proposed algorithm, classification among the three QAM types of interest is performed in two stages. The first stage includes discrimination between QAM I and QAM III as one set and QAM II as a second set. This stage is based on counting the number of phases in the normalized phase histogram, $\dot{H}_{\Phi}(\varphi)$, as follows. The maximum element in the normalized phase histogram, $\dot{H}_{\Phi}(\varphi)$, is compared with a predefined threshold, $T_{1}$. If the maximum element in the normalized phase histogram exceeds the threshold, it will be considered as a phase state. The maximum element and its two adjacent elements are nullified in order not to be counted once more. Then, the previous operation is applied to the next maximum element. Finally, the decision about the modulation type follows the rule shown in the following equation.

$$
\text { Decision }= \begin{cases}Q A M \quad \text { I or } Q A M \text { III } & \text { if } P \leq 9 \\ Q A M \quad \text { II } & \text { if } P>9\end{cases}
$$

where $P$ is the number of the elements in the normalized phase histogram that exceed the threshold.

As a result QAM II can be distinguished from QAM I and QAM III. The second stage which is concerned with the discrimination between QAM I and QAM III is accomplished using the normalized amplitude histogram, $\dot{H}_{A}(a)$, as follows. The maximum element in the normalized amplitude histogram, $\dot{H}_{A}(a)$, is compared with a predefined threshold, $T_{2}$. If the maximum element in the normalized amplitude histogram exceeds such threshold, it will be considered an amplitude state. The maximum element and its two adjacent elements are nullified in order not to be counted once more. Then, the previous operation is applied to the next maximum element. Finally, the decision about the modulation type follows the rule shown in the following equation.

$$
\text { Decision }= \begin{cases}Q A M I & \text { if } A<3 \\ Q A M \text { III } & \text { if } A \geq 3\end{cases}
$$

where $A$ is the number of the elements in the normalized amplitude histogram that exceed the threshold. 


\section{Thresholds Determination and Performance Evaluation}

In this algorithm, it is required to determine two threshold values; 1) phase threshold, $T_{1}$, used in the normalized phase histogram and 2) amplitude threshold $T_{2}$, used in the normalized amplitude histogram. Thresholds determination is obtained from 100 realizations, each with 2048 samples, for each type of interest at the SNR of 20 and $15 \mathrm{~dB}$. The QAM signals are generated with symbol rate of $10 \mathrm{kHz}$ and a sampling rate of $100 \mathrm{kHz}$. A white Gaussian noise with power spectral density of $N_{0} / 2$ is simulated and added to the signal. The fading effect, $F$, can be generated as the product of a random variable having Rayleigh distribution and the exponential of a uniformly distributed random variable [7]. It is assumed that the fading effect does not cause a significant variation of the channel gain over the signal frame considered in the proposed algorithm.

The SNR here is defined as the ratio of the received faded signal power to the noise power within the signal bandwidth. The normalized phase and amplitude histograms of the 100 realizations are trained along the interval $[0,1]$. The optimum threshold is the value that yields the highest average success rate over each type of interest at SNR of 20 and $15 \mathrm{~dB}$.

\section{Optimum thresholds}

The results associated with the determination and optimization of the phase and the amplitude thresholds are presented in Fig. 3 and Fig. 4, respectively. These figures show the overall success rate over different SNRs (20 and $15 \mathrm{~dB}$ ) at different threshold values. Fig. 3 contains three curves: 1) the dashed curve represents the average success rate for the QAM-16 types that has eight phase states (QAM I and QAM III), 2) the dotted curve represents the average success rate for the QAM-16 type that has twelve phase states (QAM II), and 3) the solid curve represents the overall average success rate. Fig. 4 shows the overall success rate over different SNRs (20 and $15 \mathrm{~dB}$ ) at different threshold values. The figure contains three curves: 1) the dashed curve represents the average success rate for the QAM-16 type that has two amplitude states (QAM I), 2) the dotted curve represents the average success rate for the QAM-16 type that has four amplitude states (QAM III), and 3) the solid curve represents the overall average success rate. 


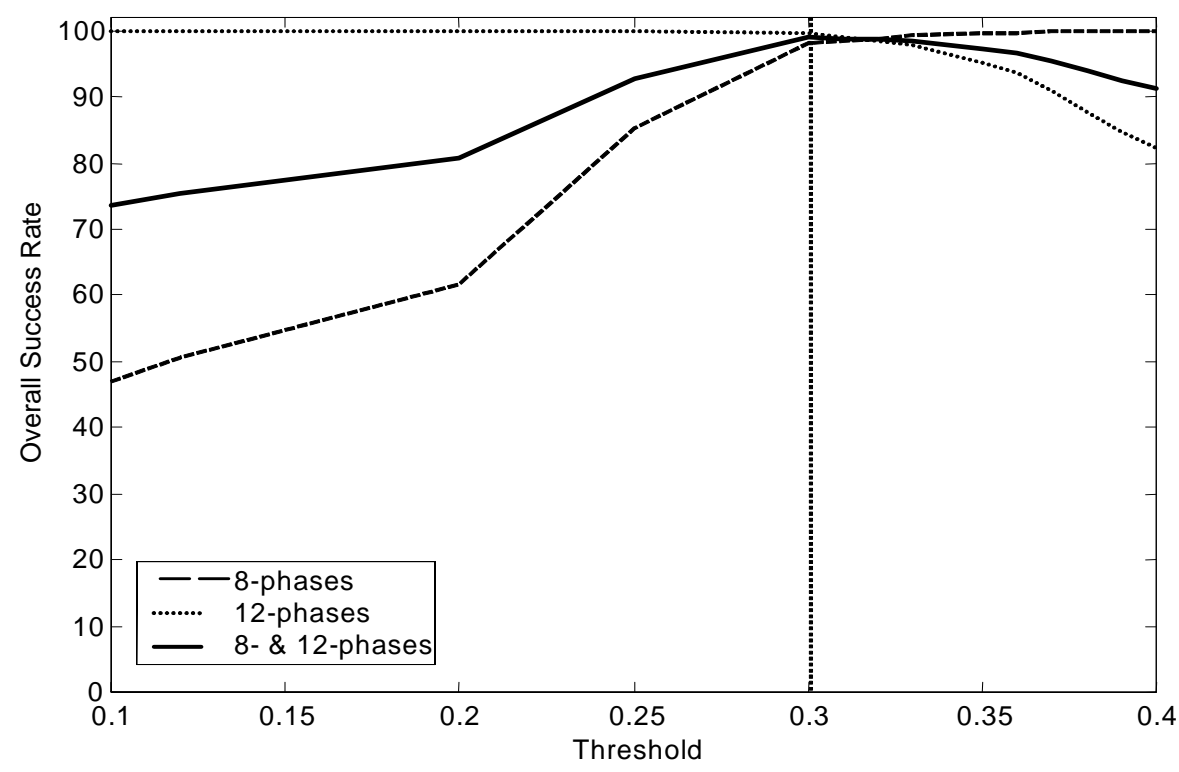

Fig. 3 Dependence of overall success rates for QAM-16 signals on phase threshold values

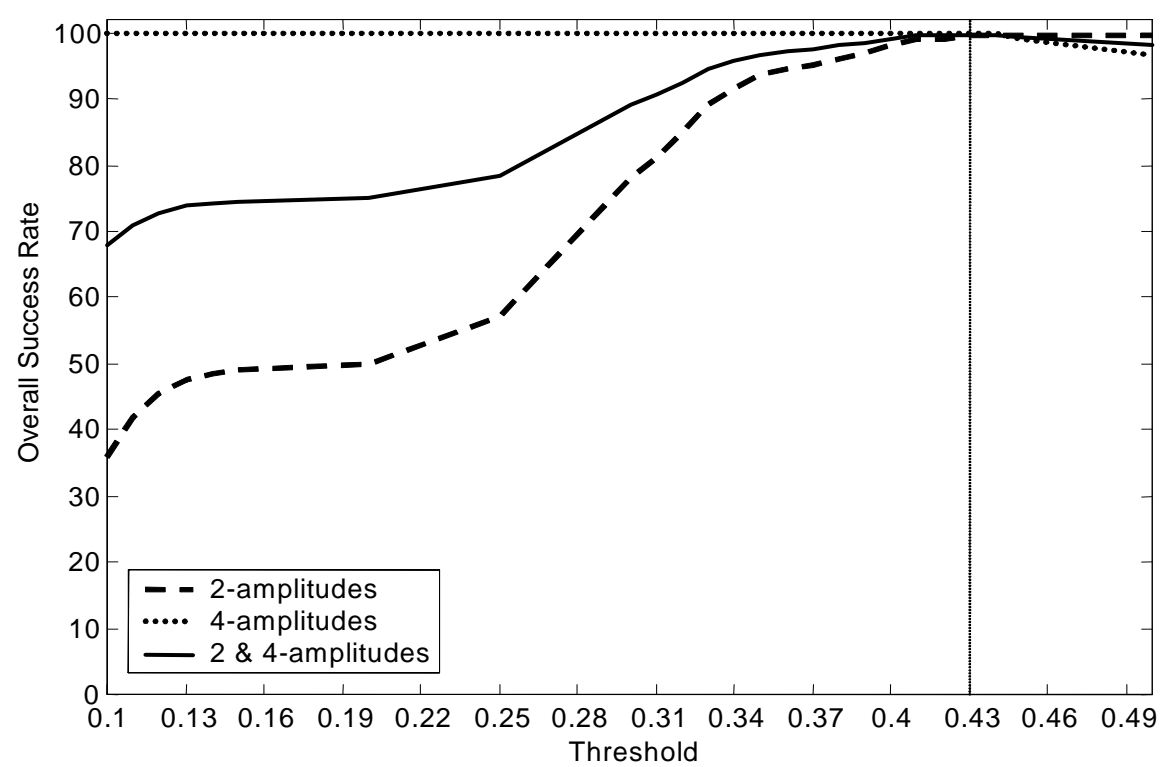

Fig. 4 Dependence of overall success rates for QAM-16 signals on amplitude threshold values

From Fig. 3, it is clear that the optimum phase threshold value, $T_{1}$, for the discrimination between QAM I and QAM III as one set and QAM II as another set is 0.3. This threshold achieves an overall success rate $>98.00 \%$. From Fig. 4, it is clear that the optimum amplitude threshold value, $T_{2}$, for the discrimination between QAM I and QAM III, based on 
the number of amplitude states, lies in the interval [0.43, 0.44]. This threshold achieves an overall success rate $>99.00 \%$ and a success rate greater than $98.50 \%$ for each set.

The evaluation of the proposed algorithm is taken from 1000 different realizations each with 2048 samples. All the results associated with the evaluation of the proposed method for QAM classification are presented in Table 2 and Table 3 for SNR of 20 and 15 respectively.

Table 2: Confusion matrix for the QAM-16 classifier at SNR $=20 \mathrm{~dB}$

\begin{tabular}{|c|c|c|c|}
\hline \multirow{2}{*}{$\begin{array}{c}\text { Simulated Modulation } \\
\text { Type }\end{array}$} & \multicolumn{3}{|c|}{ Deduced Modulation Type } \\
\cline { 2 - 4 } & QAM I & QAM II & QAM III \\
\hline QAM I & $100 \%$ & - & - \\
\hline QAM II & - & $100 \%$ & - \\
\hline QAM III & - & - & $100 \%$ \\
\hline
\end{tabular}

Table 3: Confusion matrix for the QAM-16 classifier at SNR $=15 \mathrm{~dB}$

\begin{tabular}{|c|c|c|c||}
\hline \multirow{2}{*}{$\begin{array}{c}\text { Simulated Modulation } \\
\text { Type }\end{array}$} & \multicolumn{3}{|c||}{ Deduced Modulation Type } \\
\cline { 2 - 4 } & QAM I & QAM II & QAM III \\
\hline QAM I & $91.01 \%$ & $0.00 \%$ & $8.99 \%$ \\
\hline QAM II & $0.10 \%$ & $99.20 \%$ & $0.70 \%$ \\
\hline QAM III & $1.3 \%$ & $0.10 \%$ & $98.60 \%$ \\
\hline
\end{tabular}

From the results shown in table 2 and table 3, it is clear that all digital modulation types of interest have been correctly classified with a success rate $100 \%$ at SNR $=20 \mathrm{~dB}$ and a success rate $\geq 91 \%$ at $\mathrm{SNR}=15 \mathrm{~dB}$. The proposed classifier is tested at lower value of SNR $(<15 \mathrm{~dB})$. The results show that at lower values of SNR $(14 \mathrm{~dB})$ the proposed classifier can discriminate between QAM I and QAM III types with a low success rate but gives a high success rate for QAM II as shown in table 4. Table 5 shows the overall success rates for the proposed classifier at different values of SNR. The overall success rate is calculated as the average of the success rates of the modulation types of interest. It can be shown that for an SNR $<13 \mathrm{~dB}$, the performance of the proposed classifier begins to degrade. Also, it is worth noting that QAM III is the best modulation type from the point of view of communication.

Table 4: Confusion matrix for the QAM-16 classifier at SNR $=14 \mathrm{~dB}$

\begin{tabular}{|c|c|c|c||}
\hline \multirow{2}{*}{$\begin{array}{c}\text { Simulated Modulation } \\
\text { Type }\end{array}$} & \multicolumn{3}{|c|}{ Deduced Modulation Type } \\
\cline { 2 - 4 } & QAM I & QAM II & QAM III \\
\hline QAM I & $87.91 \%$ & $0.40 \%$ & $11.69 \%$ \\
\hline QAM II & $0.00 \%$ & $99.30 \%$ & $0.70 \%$ \\
\hline QAM III & $2.20 \%$ & $0.30 \%$ & $97.50 \%$ \\
\hline
\end{tabular}


Table 5: Overall success rate at different SNR's

\begin{tabular}{|c|c|}
\hline SNR $[\mathrm{dB}]$ & $\begin{array}{c}\text { Overall Success Rate } \\
(\%)\end{array}$ \\
\hline 20 & 100 \\
\hline 15 & 96.27 \\
\hline 14 & 94.90 \\
\hline 13 & 82.35 \\
\hline 12 & 52.81 \\
\hline 11 & 37.66 \\
\hline 10 & 33.63 \\
\hline
\end{tabular}

Fig. 5 shows the amplitude histograms and the phase histograms of each signal of interest (QAM I, QAM II, and QAM III) at SNR=10 dB, each with 2048 samples. The functional flow chart for the proposed classifier is shown in Fig. 6.

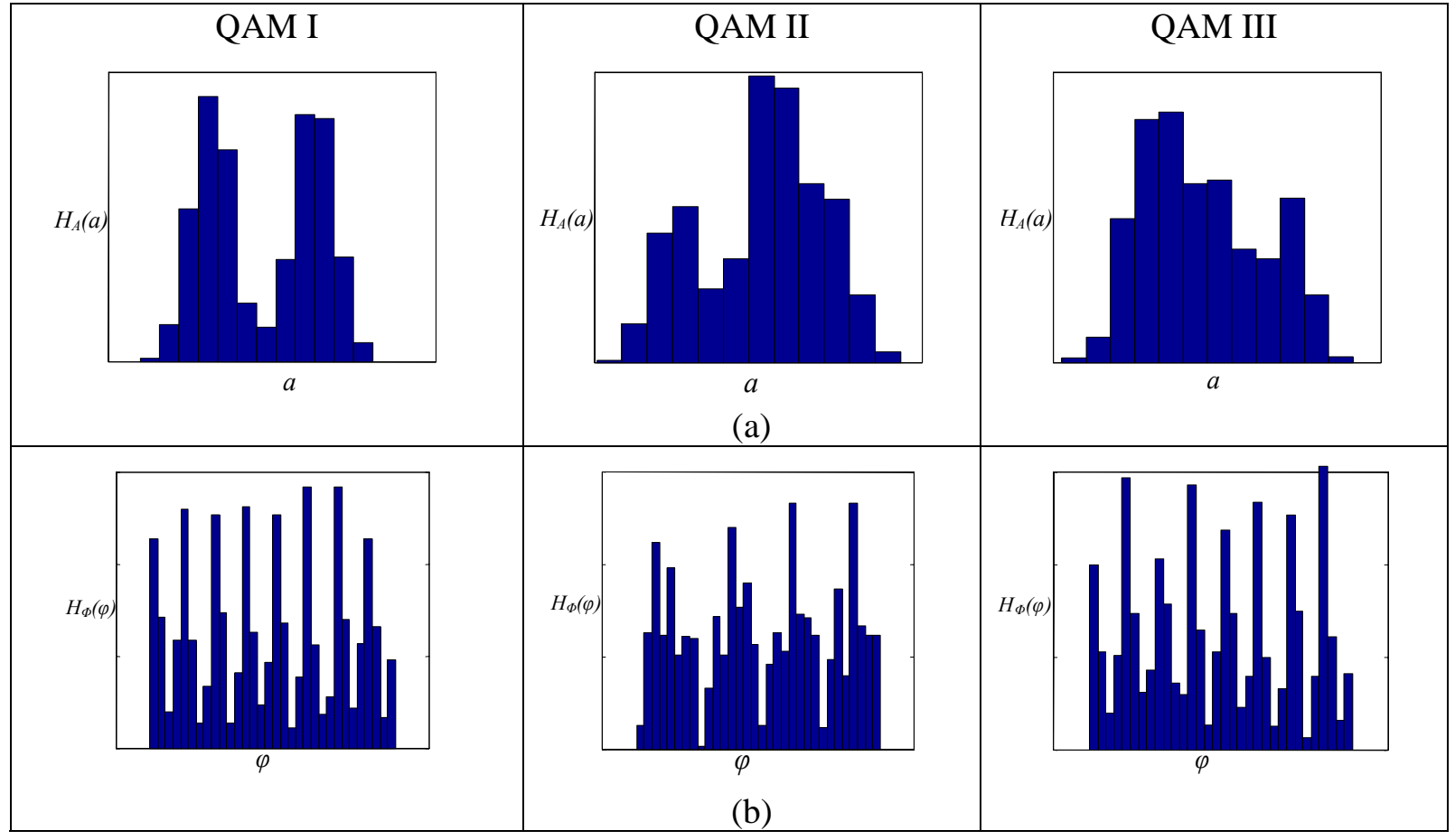

Fig. 5 a) Amplitude histograms, b) Phase histograms of the received QAM I, QAM II, and QAM III signals at $\mathrm{SNR}=10 \mathrm{~dB}$ 


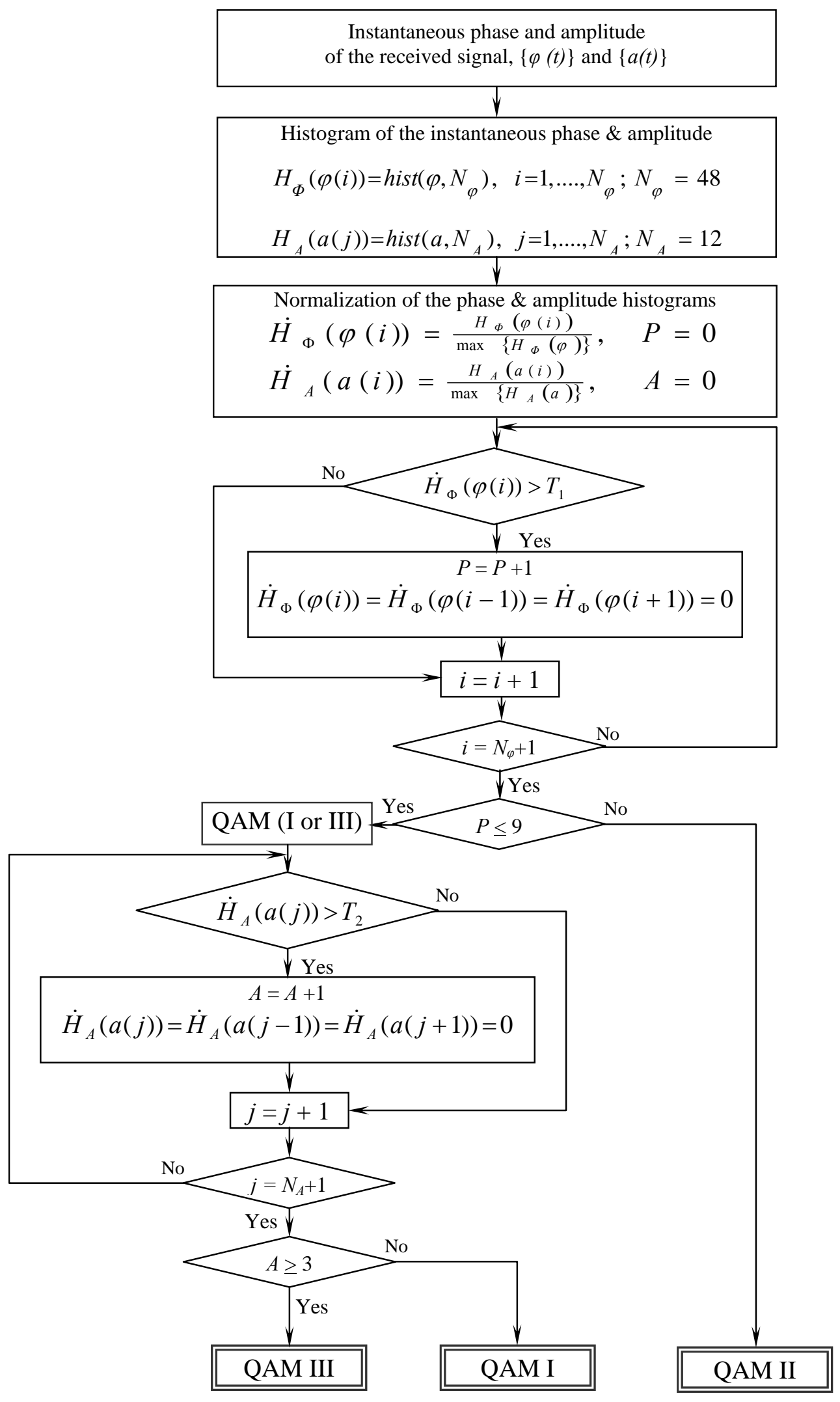

Fig. 6 The functional flow chart for the proposed 


\section{Conclusions}

In this paper, an algorithm for automatic modulation classification of 16-ary Quadrature Amplitude Modulated (QAM-16) signals has been presented. The algorithm has taken into consideration the problems due to channel impairments such as slow fading and noise. The proposed algorithm has shown good classification results and a robust behavior against the mentioned impairments. We have successfully solved the problem for three different modulation types. The algorithm seems easily extendable to include higher levels (probably with some small modifications of the key features extraction and the classification stages).

\section{References}

[1] M. Richterova, "Signal modulation recognition based on method of artificial networks", Progress in Electromagnet. Research Symposium, Hangzhou, China, Aug. 2005

[2] V. matic and V. Tadic, "Modulation classification using the power spectrum of detected signals”, Comm. System Network, CSN, benidorm, Spain, Sept. 2003.

[3] A. Swami and B. Sadler, "Hierarchical digital modulation classification using cumulants", IEEE Trans. on Communications, vol. 48, no. 3, March 2000, pp. 416-429.

[4] W. Wei and J. Mendel, "Maximum likelihood classification for digital amplitude-phase modulations”, IEEE Trans. on Communications, vol. 48, no. 2, Feb. 2000, pp. 189-193.

[5] Ahmed E. El-Mahdy, "Classification of MFSK signals over time varying flat correlated fading channels under Class-A impulsive noise”, IEE proc. on Communications, vol. 151, no. 6, December 2004, pp. 619-626.

[6] Jon G. Proakis, “Digital Communications”, New York: Mc Graw-Hill, 2000.

[7] T. Helaly, "Interception of Satellite Communication Signals”, M.Sc. thesis, MTC, Cairo, Egypt, 2001. 